\title{
Can you tell me about medication?
}

\author{
TERENCE L MOORE, MD, FRCPC
}

T

HE PATIENT WITH INFLAMMATORY bowel disease (IBD) approaches decisions about medical treatment with a healthy skepticism. The diagnosis of IBD has been delayed weeks to months while abundant (and sometimes disabling) symptomatology is ascribed, first to an infectious etiology, and then (with negative cultures) to a functional disorder of the bowel, with strong implication that the patient is coping badly with life's stresses. After a series of invasive, and sometimes uncomfortable, investigations lead to a diagnosis of IBD, the patient has to rationalize affliction with a chronic disease, the cause of which is unknown. When the understandably pessimistic patients with newly diagnosed IBD are advised that their treatment will be symptomatic, ie, that when they are quite sick they will get a lot of treatment and when they are quite well they will get none, discouragement is likely to increase, at times approaching despair.

It is extremely important in setting up a long term treatment plan for this chronic disease that the physician shows the good confidence that comes from a full understanding of the medical therapies available and transfers this confidence to the patients by careful discussion of the treatment options. It is equally important that the patients understand the effects and the side effects of the treatment used so that in partnership with their physician, they can come to the right conclusions about the effectiveness of their treatment.

Medical treatment never cures IBD, but it is dramatically effective. Thirty years ago the 10-year cumulative survival rate with both ulcerative colitis and Crohn's disease was under $80 \%$; now it is over $95 \%$ and differs minimally from survival in the general population (1). Equally important, but much harder to measure, is the dramatic impact on patient symptoms. The physician will believe that a medical therapy which lowers disease activity index is reducing the inflammatory process in the bowel. The patient with IBD will come to know that the therapies which reduce disease activity reduce pain and suffering. Those therapies which have proven impact on
Division of Gastroenterology, St Michael's Hospital, Toronto, Ontario

Correspondence and reprints: Dr TL Moore, Division of Gastroenterology, St Michael's Hospital, Toronto, Ontario M5B IW8 disease activity and which, alone or in concert, are the present treatment for patients with IBD, are reviewed here. Newer drugs, which have already had a proven impact on disease activity in clinical trials and are now being introduced into everyday therapy, will also be briefly acknowledged.

\section{STEPS IN THE SYMPTOMATIC TREATMENT OF IBD}

The investigations of a patient with newly diagnosed IBD are designed to determine the disease severity (both the extent and the activity of the disease process). Treatment decisions reflect that severity. Because disease severity changes dramatically (and usually unpredictably) it is good therapeutic strategy from the first visit to decide with the patient not only what the present treatment will be, but also what changes in treatment will be available as the disease responds (or worsens). All patients with IBD should receive advice on nutrition; most will require long periods of treatment with modestly active, but minimally toxic, 5-aminosalicylic acid (5-ASA) compounds; many will benefit from short courses of very active, but also very toxic, corticosteroid preparations; and a few will either not tolerate or fail to 


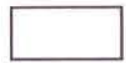

Nutrition

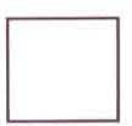

5-ASA

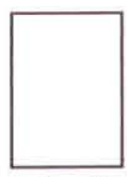

Steroids

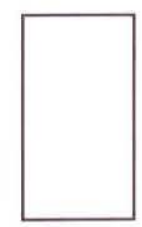

Steroid

"Alternatives"

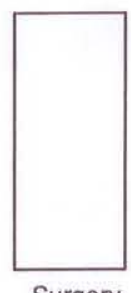

Surgery
Disease Severity

Figure 1) Steps in the symptomatic treatment of inflammatory bowel disease

respond to steroids and may benefit from steroid 'alternatives', if surgical treatment of their disease is still not indicated (Figure 1). All patients will climb up and down these treatment steps many times as the severity of their IBD fluctuates from year to year.

\section{NUTRITION}

By the time IBD is diagnosed, most patients already have some nutritional deficiencies. Those patients whose disease is severe enough to require hospitalization usually have experienced weight loss (two-thirds of Crohn's disease patients; one-half of ulcerative colitis patients), with growth failure in one-third of the pediatric age group (2). Of the many possible causes for this malnutrition, by far the most important is food avoidance by the patient who realizes that symptoms are often worse after eating (sitophobia).

It has been standard practice to incorporate elimination diets into the medical management of IBD. For example, patients with colitis are advised to avoid milk and ice cream; patients with Crohn's disease of the small bowel who have pain after eating benefit from a restricted fibre diet. The concept of 'resting the bowel' gained greater credibility when it was shown in clinical trials that an elemental enteral diet was as effective as mid-range steroid treatment in helping to remit Crohn's disease over two- or four-week periods. And 'total bowel rest', with total parenteral nutrition (TPN), remitted Crohn's disease in $60 \%$ of cases (3). Nutritional recovery was virtually assured with comprehensive enteral or parenteral feeding because adequate calories could be guaranteed. But the concept of 'bowel rest' was clearly flawed. Patients with colitis (Crohn's disease or ulcerative colitis) were less likely to remit their disease and patients who went into remission usually relapsed when they returned to a normal diet.

It is now recognized that bowel rest can be equated to bowel atrophy - that the bowel lining, especially in the large bowel, thins and works less effectively when nutrients (food) no longer are exposed to it. The mechanisms of this atrophy are complex, involving local changes in nerves, hormones and the immune system. This is forcing dramatic changes in the thinking about nutrition in IBD treatment. Food is being thought of much like drug therapy, ie, high amounts of specific nutrition can have a pharmacological effect on bowel inflammation. Studies by Greenberg (4) clearly showed that defined formula diets, taken by mouth, were as efficient as TPN in remitting acute Crohn's disease, emphasizing that complete bowel rest was not necessary. Furthermore, studies with short chain fatty acids (produced when colonic bacteria ferment fibre), fish oils, food nucleotides, etc, are not only expanding knowledge of bowel nutrition but also unravelling the mysteries of bowel inflammation and the body's immune responses.

For the present, nutrition is used primarily to replete nutritional deficits. In the future 'food pharmacology' will be shown to reduce disease activity and will be a cornerstone in the ambulatory management of patients with IBD.

\section{5-AMINOSALICYLIC ACID}

Sulphasalazine (Salazopyrine; Pharmacia) was used to treat active colitis for 40 years before its efficacy was confirmed for both ulcerative colitis (5) and Crohn's colitis (6). Sulphasalazine is also being used to treat Crohn's disease of the small bowel, even though efficacy cannot be considered proven. Classic British clinical trials proved the usefulness of sulphasalazine in maintaining remissions of ulcerative colitis in the ' $60 \mathrm{~s}(7,8)$. But sulphasalazine has not shown benefit in maintaining remissions of Crohn's disease (6). The use of sulphasalazine has been limited by side effects (nausea, interference with food folate absorption, suppression of sperm counts, hemolysis, etc) in $15 \%$ of patients. The studies of Azad Khan demonstrated that when sulphasalazine was cleaved to its two metabolites (5-ASA and sulphapyridine, 5-ASA was the active moiety and sulphapyridine was considered to be an inactive carrier (9). Fortunately, the sulphapyridine moiety was responsible for most of the side effects (Figure 2).

Topical 5-ASA (given by suppository or enema) was shown to be effective in the treatment of active distal colitis (10), even in patients intolerant to sulphasalazine, and is now the initial treatment of choice for patients with active colitis confined to the left side of the colon (11). But patients with inflammation higher up in the colon or in the distal small bowel require an oral preparation. This initially presented a problem as $5-\mathrm{ASA}$ is readily absorbed in the proximal small bowel and unavailable for more distal, topical effect. Fortunately the pharmaceutical industry responded to this challenge by devising delivery systems for oral 5-ASA that release maximal concentrations of 5-ASA along the small bowel (Pentasa; Nordic), in the ileocecal area (Salofalk; Interfalk, Mesasal; SmithKline Beecham), or colon (Asacol; Norwich Eaton, Dipentum; Pharmacia).

There is not yet agreement on how topical 5-ASA works to block inflammation in the bowel wall. It may block prostaglandin synthesis or scavenge oxygen free radicals in the bowel wall, 
or inhibit leukotriene formation by blocking the metabolism of arachidonic acid in white blood cells (12). To deliver maximal concentrations of 5 ASA to the colon, the drug is coated with an acrylic polymer (Asacol, Salofalk, Mesasal) or linked to a second molecule of 5-ASA (Dipentum) releasing the drug into the distal ileum and colon. About half of the drug is absorbed and can rarely give rise to those side effects attributable to salicylates (headache, rhinitis, urticaria, asthma, hair loss, etc). The dimeric (double molecule) 5-ASA drug (Dipentum) is much more likely $(15 \%)$ to produce diarrhea than the other oral 5-ASA preparations $(4 \%)$, because of an effect on small bowel secretion unique to that dimeric formulation.

There is almost universal agreement on the effectiveness of the new oral 5-ASA drugs in treating first attacks of ulcerative colitis (13) and in maintaining remissions of ulcerative colitis. Oral 5-ASA preparations have equivalent effectiveness to sulphasalazine with fewer side effects, but are more than twice as expensive. They have already become the drugs of choice in patients with diffuse, mildly active or quiescent ulcerative colitis who are intolerant to sulphasalazine.

It is much harder to prove drug efficacy in Crohn's disease than it is in ulcerative colitis. Clinical remission from Crohn's disease is very difficult to define and even more difficult to achieve. It is becoming obvious from uncontrolled clinical trials that higher doses of oral 5-ASA (up to $5 \mathrm{~g}$ per day) will be needed to remit Crohn's disease. Hopefully, the development of new drug delivery systems to achieve maximal concentrations higher in the small bowel (Pentasa) will prove more useful in patients whose active Crohn's disease is located more proximally in the small bowel or in the stomach. An international study group (15) has reported that patients with Crohn's disease in remission are less likely to exacerbate their disease when treated with $1.5 \mathrm{~g}$ per day of Claversal (SmithKline Beecham) for up to one year $(22.4 \%$ relapse rate versus $36.2 \%$ in placebotreated controls). But the evidence is

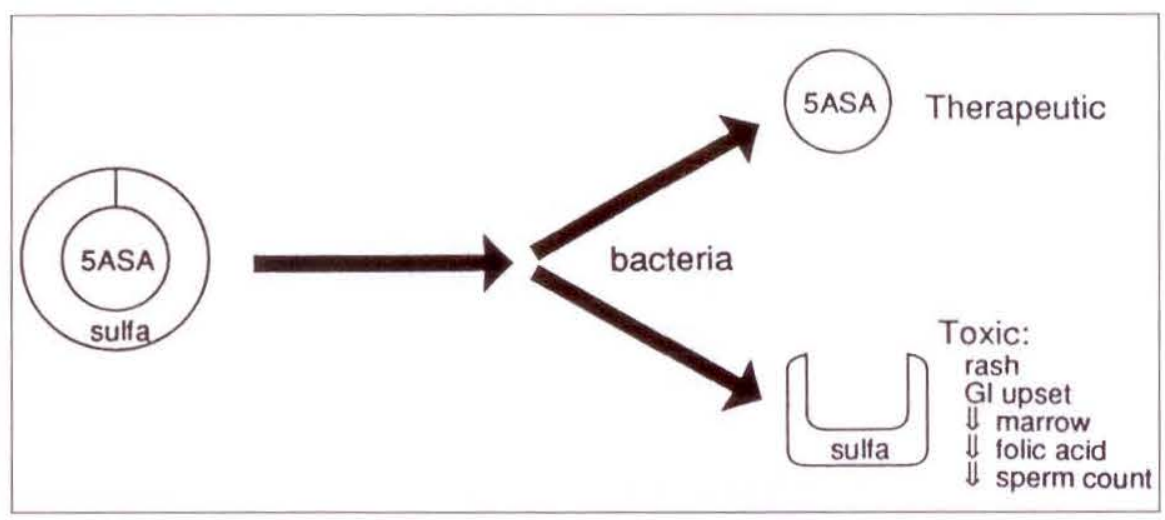

Figure 2) Sulphasalazine and its component metabolites - the therapeutic 5-aminosalicylic acid (5-ASA) moiety, and the inactive, toxic sulpha moiety sulphapyridine

not yet convincing enough to use oral 5-ASA routinely in the prophylaxis of 'quiescent' Crohn's disease.

With better delivery systems and dosing schemes, proven by controlled clinical trials and with improved understanding of drug action on gut inflammation, the future of oral 5-ASA drugs in the treatment of Crohn's disease is assured. The future is already here in the treatment of ulcerative colitis with 5-ASA.

\section{STEROIDS}

Steroids are effective in the treatment of IBD. Within days of starting mid-range prednisone ( 30 to $40 \mathrm{mg}$ per day) patients experience an improved sense of well-being with return of appetite and an increase in energy. By two weeks, drug action to control inflammation in the bowel is peaking, symptoms are receding and the gradual withdrawal of the drug can usually be commenced. The proof that steroids (cortisone) work in acute ulcerative colitis came within two years of their introduction in the early ' 50 s (16). And the development of synthetic steroids (eg, prednisolone) reduced toxicity and permitted topical use (as enemas, suppositories and rectal foams) depending on the extent of the colitis (17). The use of corticosteroids in active Crohn's disease is similarly effective with about three-quarters of patients remitting their disease, clinically (6) but not endoscopically (18). As with ulcerative colitis, steroids fail to maintain remissions of Crohn's disease; chronic use of steroids, even in low doses, is to be avoided in patients whose IBD is in clinical remission.

Everybody knows that steroids are toxic drugs. Patients given mid-range prednisone often experience irritability, depressive reaction and sleeping difficulties. If the disease does not remit as anticipated and the dose of prednisone is continued, the patient shows visible side effects (facial swelling, easy bruising, elevated blood pressure, etc). There are also concerns about less visible complications, such as adrenal suppression, cataracts, myopathy, avascular necrosis of the hip joints, etc. Even with topical steroid enemas given chronically, there is sufficient absorption into the body to give these systemic side effects.

And so, while everybody uses steroid medications in the acute exacerbations of diffuse IBD, the development of less toxic steroid drugs is eagerly awaited. Both tixocortol pivalate and budesonide, given by enema, are as effective in treating distal ulcerative colitis as betamethasone or hydrocortisone enemas $(19,20)$. Although the drugs are absorbed, they are rapidly cleared by the liver and kidneys, dramatically reducing systemic toxicity. Their use in treating distal colitis is presently limited by availability (and the success with 5 . ASA enemas). Because steroids have this topical activity in the gut, it is hoped that new steroids will be developed that can be taken by mouth, can have topical activity and, by being either poorly absorbed or rapidly excreted, can be free from the side effects 
TABLE 1

Changing medical treatment of inflammatory bowel disease

\begin{tabular}{|c|c|c|}
\hline & 'Old' treatments & 'New' treatments \\
\hline Nutrition & Elimination diets & Enteral formulas, short chain fatty acids \\
\hline $\begin{array}{l}\text { 5-Aminosalicylic } \\
\text { acid }\end{array}$ & Sulphasalazine & $\begin{array}{l}\text { Asacol }{ }^{(1)} \text {, Dipentum } \\
\text { Pentasa }^{(2)} \text {, Salofalk }\end{array}$ \\
\hline Steroids & Prednisone & Tixocortil, budesonide \\
\hline Immunosuppressives & $\begin{array}{l}\text { 6-Mercaptopurine, } \\
\text { azathioprine }\end{array}$ & Cyclosporine, methotrexate \\
\hline
\end{tabular}

Registerd trademark

which limit the use of this dramatically effective group of drugs.

\section{STEROID ALTERNATIVES}

Most patients who are acutely ill with extensive IBD respond to steroids given in a decreasing dose (beginning $40 \mathrm{mg}$ of prednisone) over a short period of time (two to three months). The steroid toxicity is acceptable and less toxic drugs (usually 5-ASA) can then be used to maintain clinical improvement. But some of these sick patients (up to 20\%) are steroid resistant and face certain toxicity from long term use of steroids. For these patients, the steroid 'alternatives' have already begun to offer safer and more effective medical treatment.

6-Mercaptopurine and azathioprine: Thanks largely to the persistence of a group of New York gastroenterologists, 6-mercaptopurine (6-MP) is the best studied and most acceptable steroid alternative. Azathioprine (Imuran; Burroughs Wellcome), an active metabolite of 6-MP, has comparable clinical efficacy. In both ulcerative colitis and Crohn's disease (especially when it involves the colon), remission rates of 50 to $70 \%$ can be expected. By using a relatively low drug dosage of $1 \mathrm{mg} / \mathrm{kg}$ bodyweight, most of the toxicity of these drugs is predictable and reversible. Pancreatitis occurs in 3\% of cases between three and four weeks of starting treatment, and reverses without permanent damage when the drug is stopped. Bone marrow suppression (2\%) can be avoided by monitoring peripheral blood counts and lowering drug dosage if counts fall. The development of opportunistic infections with immunosuppression is less than with prednisone. Although the drugs are mutagenic, there has been no unusual occurrence of car- cinoma or lymphoma with their use (21). Although the drugs are teratogenic in higher doses, there is good evidence of their safety in pregnancy at the doses used to treat IBD (22). A therapeutic bonus in Crohn's disease has been the healing of enterocutaneous and internal fistulae in some $(50 \%)$ of the patients treated with 6-MP or azathioprine. The major limitation of the drug (apart from side effects) is the long time required for the drug to become active (3.1 months). Usually this means that the patient faces another three months of steroid toxicity, once the decision has been made to use 6-MP or azathioprine. The search for other, faster acting, steroid alternatives continues. reports that cyclosporine, an immunosuppressive drug used in organ transplantation, acts rapidly to improve steroid-refractory patients with either (25). Because the drug has frequent side effects (numbness in the limbs, inner trembling, renal toxicity, etc) it has usually been used for short periods of time (less than three months). It is likely that the drug will be maximally useful if used for longer periods of time (especially as the disease recurs rapidly when the drug is discontinued). Because of its toxicity, cyclosporine should be used in long term studies only in clinical research programs at centres experienced in the use of this drug.

Methotrexate: Methotrexate is an antimetabolite primarily used in cancer chemotherapy. When found to have anti-inflammatory properties its use was extended to several chronic diseases, such as psoriasis and rheumatoid arthritis. In small, open trials it improved both refractory ulcerative colitis and Crohn's disease in about threeCyclosporine: There are preliminary Crohn's disease or ulcerative colitis quarters of cases. Its advantages would appear to be rapid onset of action (two to eight weeks) and ease of delivery (25 $\mathrm{mg}$ by intramuscular injection once a week). Side effects (marrow suppression, gastrointestinal upset, liver damage) are significant. If the early enthusiasm for this new treatment can be confirmed in clinical trials, the results will certainly outweigh the side effects, and methotrexate will become an important alternative to steroids when quick clinical response is required in very sick patients.

Antibiotics: There can be little doubt that antibiotics are useful in treating many of the complications of IBD. Metronidazole (Flagyl; Rhône-Poulenc) given in large doses ( $500 \mathrm{mg}$ three times per day) for long periods of time (over six months) improves perianal Crohn's disease (27). Vancomycin (Vanocin; Lilly) improves ulcerative colitis and Crohn's disease of the colon when there is complicating Clostridium difficile infection (28). There is also exciting preliminary evidence that antibiotics (such as metronidazole) which have activity against the bacteria of the colon, may reduce the activity of the primary disease process. Trials are underway to see if less toxic antibiotics, more suitable for long term use (eg, norfloxacin [Noroxin; Merck Sharp Dohme]) may be useful in treating IBD.

\section{SUMMARY}

The medical treatment of IBD is changing (Table 1). Elimination diets are giving way to dietary supplements to nourish the bowel and reduce inflammation. The frequently toxic component of sulphapyridine has been removed to produce the new 5-ASA preparations (and the vehicle replacing the sulpha moiety permits targeting of maximal drug concentrations all along the bowel). New, topically active steroids are clearly less toxic than glucocorticoids in present use and seem to be as effective. New immunosuppressive and anti-inflammatory drugs are producing useful alternatives to steroid therapy. In the past 30 years, the lethal impact of IBD has been removed; in the next 10 years, pain and suffering will be dramatically reduced. 


\section{REFERENCES}

1. Bayless TM. Prognosis of Idiopathic inflammatory bowel disease. In: Kirsner JB, Shorter R. Inflammatory Bowel Disease. Philadelphia: Lea Febiger, 1988.

2. Seidman EG. Nutritional management of inflammatory bowel disease. Gastroenterol Clin North Am $1989 ; 18.1: 129-55$

3. Christie PM, Hill GL. Effects of intravenous nutrition on nutrition and function in acute attacks of inflammatory bowel disease. Gastroenterology 1990;99:730-6.

4. Greenberg GR, Fleming CR, Jeejeebhoy KN, Rosenberg IH, Sales D, Tremaine WJ. Controlled trial of bowel rest and nutritional support in the management of Crohn's disease. Gut 1988;29:1309-15.

5. Dick AE, Grayson MJ, Carpenter RG, et al. Controlled trial of sulphasalazine in the treatment of ulcerative colitis. Gut 1964;15:437-42.

6. Summers RW, Switz DM, Sessions JT, et al. National Cooperative Crohn's disease study: Results of drug treatment. Gastroenterology 1979;77:847-69.

7. Misiewicz JJ, Lennard-Jones JE, Connell AM, et al. Control trial of sulphasalazine in maintenance therapy for ulcerative colitis. Lancet 1965;i:185-8.

8. Dissanayake AS, Truelove SC. A controlled therapeutic trial of long-term maintenance treatment of ulcerative colitis with sulphasalazine (Salazopyrin). Gut 1973;14:923-6.

9. Azad Khan AK, Piris J, Truelove SC. An experiment to determine the active therapeutic moiety of sulphasalazine. Lancet 1977;ii:892-5.

10. Campieri M, Lafranchi GA, Bazzochi
G, et al. Treatment of ulcerative colitis with high-dose 5-aminosalicylic acid enemas. Lancet $1981 ;$ ii:270-1.

11. Sutherland LR, Martin F, Greer S, et al. 5-aminosalicylic acid enema in the treatment of distal ulcerative colitis, proctosigmoiditis and proctitis. Gastroenterology 1987;92:1894-8.

12. Robinson MG. New oral salicylates in the therapy of chronic idiopathic inflammatory bowel disease. Gastroenterol Clin North Am 1989;18:43-50.

13. Rao SS, Dundas SA, Holdsworth CD, et al. Olsalazine or sulphasalazine in first attacks of ulcerative colitis? A double blind study. Gut 1989;30:675-9.

14. Riley SA, Mani V, Goodman MJ, et al. Comparison of delayed-release 5-aminosalicylic acid (mesalazine) and sulfasalazine as mainteanance treatment for patients with ulcerative colitis. Gastroenterology 1988;94:1383-9.

15. Thomson ABR, et al. Coated oral 5 -aminosalicylic acid versus placebo in maintaining remission of inactive Crohn's disease. Aliment Pharmacol Ther 1990;4:55-64.

16. Truelove SC, Witts J. Cortisone in ulcerative colitis: Final report on a therapeutic trial. Br Med J 1955;2:1041-5.

17. Jewell DE. Corticosteroids for the management of ulcerative colitis and Crohn's disease. Med Clin North Am 1989;18:21-34

18. Modigliani R, Mary J-Y, et al. Clinical, biological, and endoscopic picture of attacks of Crohn's disease. Evolution of prednisolone. Gastroenterology 1990;98:811-8.

19. Hanauer SB, Kirsner JB, Barrett WE. The treatment of left-sided colitis with tixocortol pivalate. Gastroenterology 1986;90:1449.
20. Danielsson A, Hellers G, et al. A controlled randomised trial of budesonide versus prednisolone retention enemas in active distal colitis. Scand J Gastroenterol 1989;22;987-994

21. Present $\mathrm{DH}$, Meltzer SJ, et al. 6-Mercaptopurine in the management of inflammatory bowel disease: Short and long term toxicity. Ann Intern Med 1989;111:641-9.

22. Alstead EM, Ritchie JK, et al. Safety of azathioprine in pregnancy and inflammatory bowel disease. Gastroenterology 1990;99:443-7.

23. Peltekian KM, Williams $\mathrm{CN}$, MacDonald AS, Roy PD, Czolpinska E. Open trial of cyclosporine in patients with severe active Crohn's disease refractory to conventional therapy. Can J Gastroenterol 1988;2:5-11

24. Brynskov J, Freund L, et al. A placebo controlled, double-blind, randomized trial of cyclosporine therapy in active chronic Crohn's disease. N Engl J Med 1989;321:845-50.

25. Lichtiger S, Present DH. Preliminary report: Cyclosporine in treatment of severe active ulcerative colitis. Lancet 1990;336:16-9.

26. Kozaree RA, Patterson SJ, et al. Methotrexate induces clinical and histologic remission in patients with refractory inflammatory bowel disease. Ann Intern Med 1989;110:353-6.

27. Brandt LJ, Bernstein LH, et al. Metronidazole therapy for perianal Crohn's disease. A follow-up study. Gastroenterology 1982;83:383-7.

28. Trynka YM, Lamont JT. Association of Clostridium difficile toxin with symptomatic relapse in chronic inflammatory bowel disease. Gastroenterology 1981;80:693-6. 


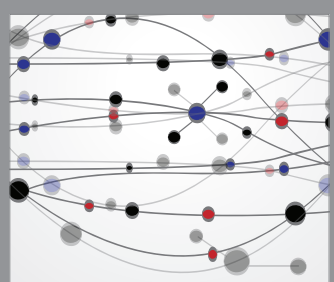

The Scientific World Journal
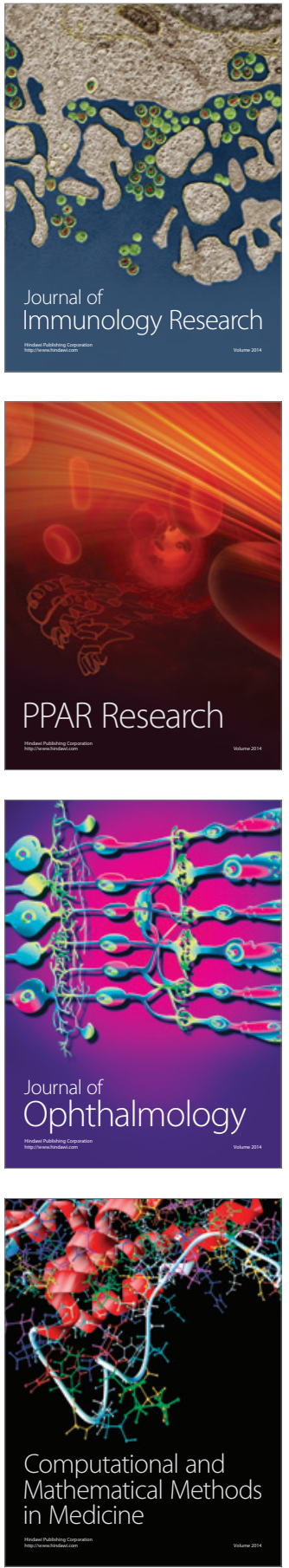

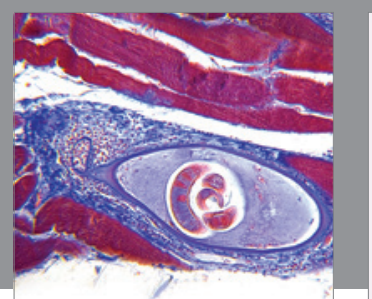

Gastroenterology Research and Practice

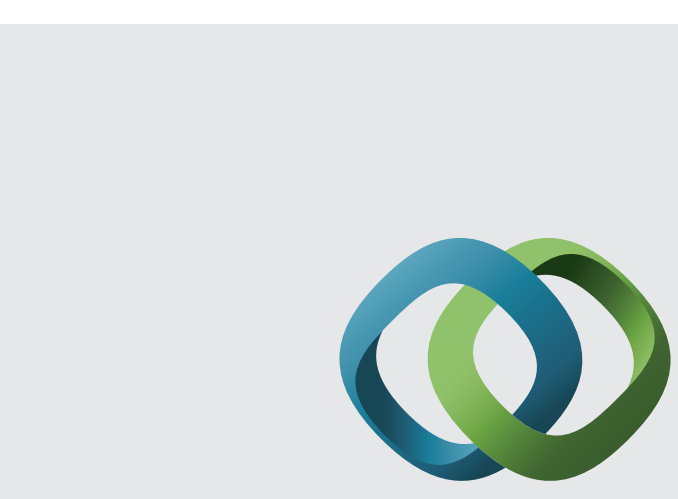

\section{Hindawi}

Submit your manuscripts at

http://www.hindawi.com
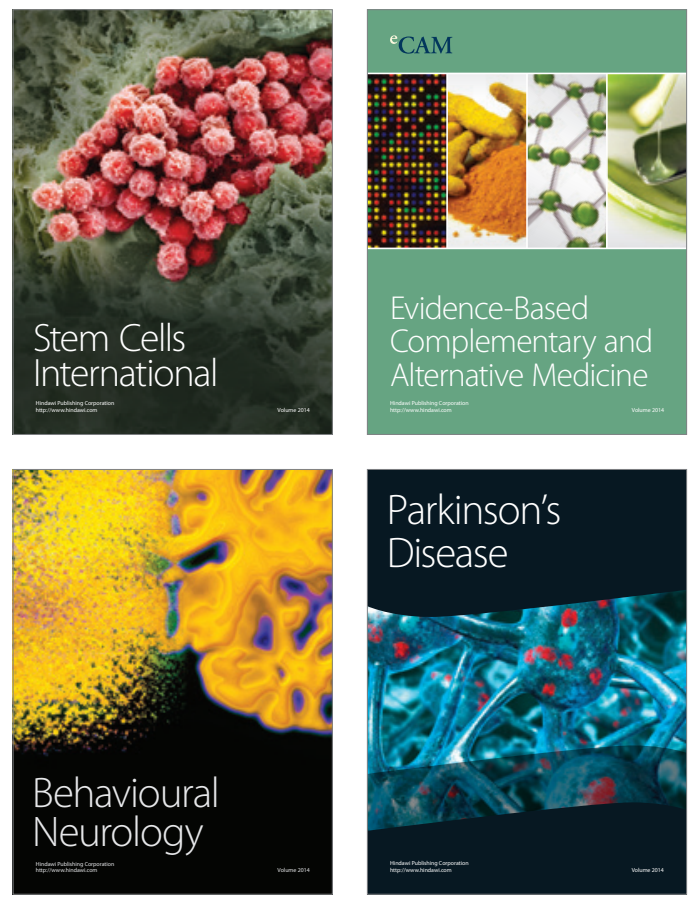
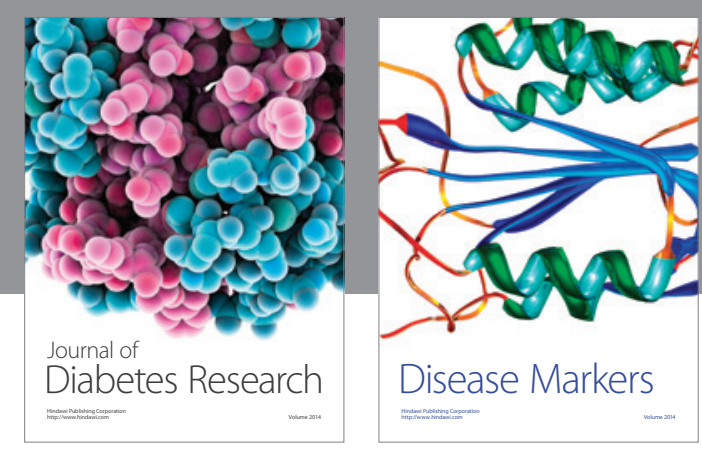

Disease Markers
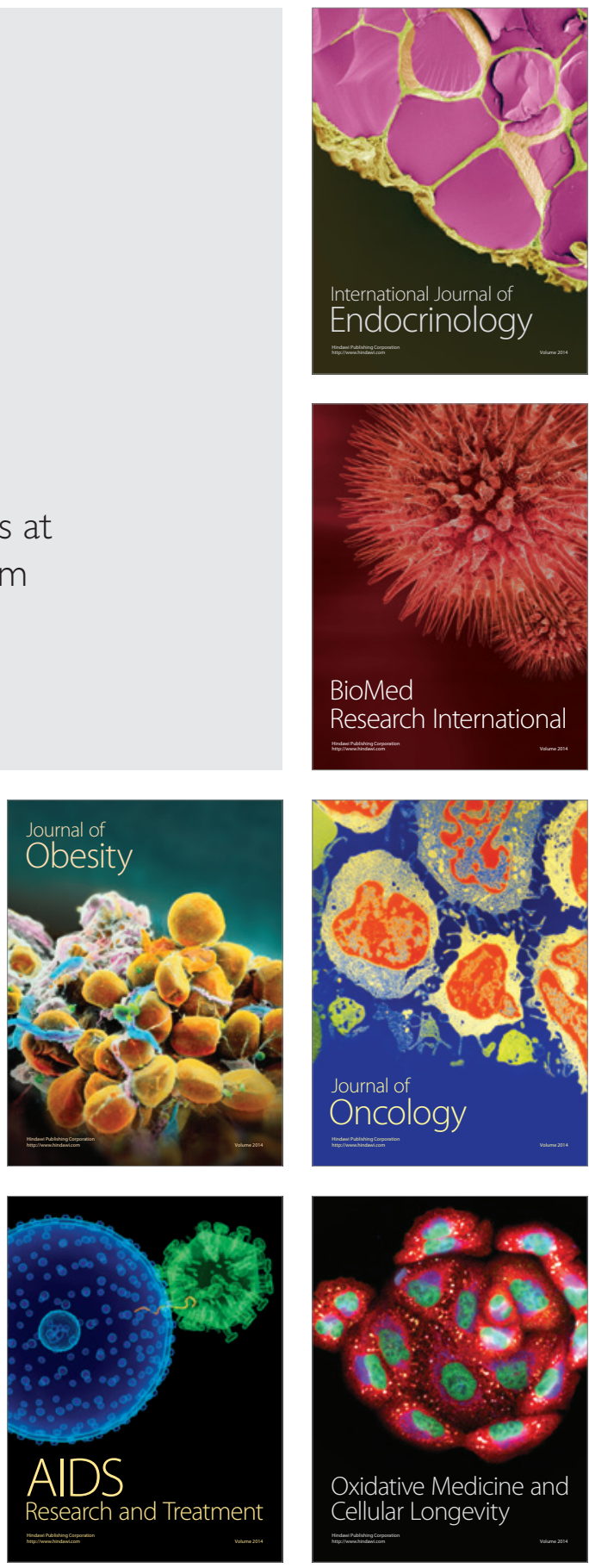